\title{
Post-operative Changes of Cerebral Glucose Metabolism in Patients with Lumbar Spinal Stenosis with Pre-operative Anxiety: Statistical Parametric Mapping Analysis of F-18 FDG Brain PET
}

\author{
Seong-Jang Kim¹, Kuen Tak Suh², Jeung II Kim³, Jong Min Lim³, Tae Sik Goh³, Jung Sub Lee ${ }^{3}$ \\ 'Department of Nuclear Medicine, Pusan National University School of Medicine, Busan, Korea \\ ${ }^{2}$ Department of Orthopaedic Surgery, Pusan National University Yangsan Hospital, \\ Pusan National University School of Medicine, Yangsan, Korea \\ ${ }^{3}$ Department of Orthopaedic Surgery, Pusan National University School of Medicine, Busan, Korea
}

\begin{abstract}
Study Design: A prospective study.
Purpose: To assess postoperative changes in cerebral glucose metabolism in anxiety patients with lumbar spinal stenosis (SS). Overview of Literature: Although an association between preoperative anxiety and abnormal cerebral glucose metabolism may exist, only a limited number of studies using F-18 fluorodeoxyglucose positron emission tomography (FDG PET) have evaluated preoperative to postoperative changes in cerebral glucose metabolism in SS patients in detail.

Methods: The present study was designed to assess preoperative to postoperative changes in cerebral glucose metabolism in anxiety patients with SS. F-18 FDG PET with statistical parametric mapping analyses was used to compare preoperative and postoperative regional brain glucose metabolism in 18 SS patients.

Results: F-18 FDG PET scans showed postoperative activation of several brain clusters in gray matter. These included left parahippocampus, left cerebellar tonsil, left inferior semi-lunar lobule, and right cerebellar tonsil. Areas that were deactivated postoperatively were the right insula, left fusiform gyrus, left orbitofrontal cortex, left inferior frontal gyrus, left middle frontal gyrus, left precuneus, and left inferior frontal gyrus.

Conclusions: SS patients with preoperative anxiety showed altered cerebral glucose metabolism at postoperative follow-up.
\end{abstract}

Key Words: Lumbar spinal stenosis, Preoperative anxiety, Postoperative change, Cerebral glucose metabolism

\section{Introduction}

Impending surgery is a stressful condition and people awaiting an operation experience anxiety [1]. Preoperative anxiety is characterized by subjective feelings of tension, apprehension, nervousness, and worry. Research has shown that preoperative anxiety is related to fears of surgical failures, anesthesia, fear of loss of control, and fear of death
[2]. It is speculated that presurgical patients display a fear/anxiety syndrome because they are fearful of surgery and are anxious about unknown consequences.

A recent study demonstrated that amygdala and insular hyperactivity is common to both social anxiety disorder and healthy subjects undergoing fear conditioning [3]. This conditioned fear provides a mechanism to explain fear and anxiety in public, and may be relevant in preoperative anxiety [4]. According to the model for conditioned fear, the amyg-

Received May 3, 2010; 1st Revised Jun 16, 2010; Accepted Jun 27, 2010

Corresponding author: Jung Sub Lee, MD

Department of Orthopaedic Surgery, Pusan National University School of Medicine,

10 Ami-dong, Seo-gu, Busan 602-739, Korea

Tel: +82-51-240-7248, Fax: +82-51-247-8395, E-mail: jungsublee@ pusan.ac.kr 
dala plays a pivotal role in the elimination of conditioned responses, and the prefrontal cortex appears to have a modulatory effect on amygdala function [5,6]. From these findings, it can be hypothesized that if patients are faced with a major operation, a stressful condition, these brain areas become dysfunctional.

On F-18 fluorodeoxyglucose positron emission tomography (F-18 FDG PET), panic disorder patients showed increased cerebral glucose metabolism in various brain areas including amygdala, hippocampus, thalamus, cerebellar tonsil, and pons [7]. However, in another study testing panic disorder patients at rest, F-18 FDG PET did not reveal glucose metabolic differences in global gray matter between patients and normal controls [8,9]. After treatment with imipramine, imipramine-treated panic disorder patients showed decreased cerebral glucose metabolism in the cerebral cortex compared with nonmedicated panic disorder patients, but the same abnormally low left and right hippocampal and posterior inferior prefrontal glucose metabolic ratios were observed as in the nonmedicated study participants.

Although a possible association between preoperative anxiety and abnormal cerebral glucose metabolism may exist, there have been a limited number of detailed studies of F-18 FDG PET in preoperative anxiety patients and postoperative changes of cerebral glucose metabolism in patients with lumbar spinal stenosis (SS). The primary purpose of the current study was to determine whether patients with SS and pre-operative anxiety show postoperative changes in cerebral glucose metabolism.

\section{Materials and Methods}

We studied 18 patients who were treated by posterior decompression with fusion using pedicle screw fixation for the surgical treatment of lumbar SS. The diagnosis of SS was based on clinical presentation and radiological findings (plain radiograph, computed tomography, and magnetic resonance [MR] imaging). All patients were diagnosed as having SS. All patients between 46 and 78 years of age presenting with SS were asked to voluntary be tested on a Hamilton anxiety rating scale (HARS) [10] and a Zung self-rating anxiety scale (ZAS) [11] and to undergo F-18 FDG brain PET imaging preoperatively and 6 months postoperatively.

All subjects were also examined clinically before entering the study to rule out any hidden metabolic disease or psychiatric disease that could affect cerebral glucose metabo- lism. In addition, brain MR imaging studies were performed in all subjects before entering the study in order to rule out any organic brain lesions. Subjects with a history of neuromuscular disease, endocrine disease, connective tissue abnormalities, organic brain disease, psychiatric disease or previous spinal surgery were excluded from the study. All subjects provided informed consent before the examinations and measurements. The study was approved by the Clinical Research Ethics Committee of the university and the hospital.

\section{F-18 FDG brain PET}

Single frame brain PET scans of 15 minute duration were acquired starting 60 minutes after intravenous injection of $370 \mathrm{MBq}$ (10 mCi) F-18 FDG using a Gemini PET/CT scanner (Philips, Milpitas, CA, USA). Scanning was done with the subjects under resting conditions with their eyes closed and ears unplugged, comfortably lying in a darkened and quiet room. Subjects fasted for at least 6 hours before positron emission tomography (PET) imaging. PET images were reconstructed using 3D RAMLA (2 repetition, 0.006 relaxation parameter) and displayed in a $128 \times 128$ matrix (pixel size $=2 \times 2 \mathrm{~mm}$, with a slice thickness of $2 \mathrm{~mm}$ ). Attenuation correction was done with a uniform attenuation coefficient $(\mu=0.096 / \mathrm{cm})$. In-plane and axial resolution of the scanner were 4.2 and $5.6 \mathrm{~mm}$ full width at half maximum (FWHM), respectively.

\section{Image analysis}

Spatial preprocessing and statistical analysis were done using the SPM2 program in Matlab ver. 7.2 (The MathWorks, Inc., Natick, MA, USA). All the reconstructed F-18FDG brain PET images were spatially normalized into Montreal Neurological Institute (MNI, McGill University, Montreal, QU, Canada) standard templates by an affine transformation (12 parameters for rigid transformations) and a non-linear transformation, then smoothed with a FWHM 8-mm Gaussian kernel to increase the signal-tonoise ratio and to account for subtle variations in anatomic structures. To remove the effects of the difference in the overall counts, the voxel counts were normalized to the mean voxel count of the gray matter in each PET image using proportional scaling.

In view of a priori knowledge suggesting involvement of the cingulate, hippocampus, inferior frontal cortex, and 
striatum in the anxiety disorders, an uncorrected $p$-value of $p<0.001$, was chosen for the analysis of these regions in order to minimize type I errors. The clusters that passed this threshold were considered significant at $p<0.05$ corrected for multiple comparisons using a false discovery rate.

The Talairach brain coordinates were estimated from a non-linear transformation from MNI space to Talairach space (ver. 1.1, Talairach Daemon Client, Research Imaging Center, University of Texas Health Science Center at San Antonio). For the visualization of the t-score statistics (SPM $\{t\}$ map), significant voxels were projected onto the 3-dimensional rendered brain or a standard high-resolution

Table 1. Activated brain areas at follow-up F-18 FDG PET study in spinal stenosis patients with preoperative anxiety

\begin{tabular}{ccccccll}
\hline \hline & & \multicolumn{3}{c}{ Coordinates } & & & \\
\cline { 3 - 5 } Cluster size & Hemisphere & $\mathrm{X}$ & $\mathrm{Y}$ & $\mathrm{Z}$ & Peak Z & p-value & Structure \\
\hline 20 & Left & -14 & -44 & -46 & 3.35 & $<0.001$ & Cerebellar tonsil \\
12 & Left & -30 & -20 & -22 & 3.4 & $<0.001$ & Parahippocampus \\
11 & Left & -22 & -68 & -50 & 3.32 & $<0.001$ & Cerebellar inferior semi-lunar lobule \\
6 & Right & 24 & -54 & -50 & 3.21 & 0.001 & Cerebellar tonsil \\
\hline
\end{tabular}

F-18 FDG PET: F-18 fluorodeoxyglucose positron emission tomography, BA: Brodmann area.
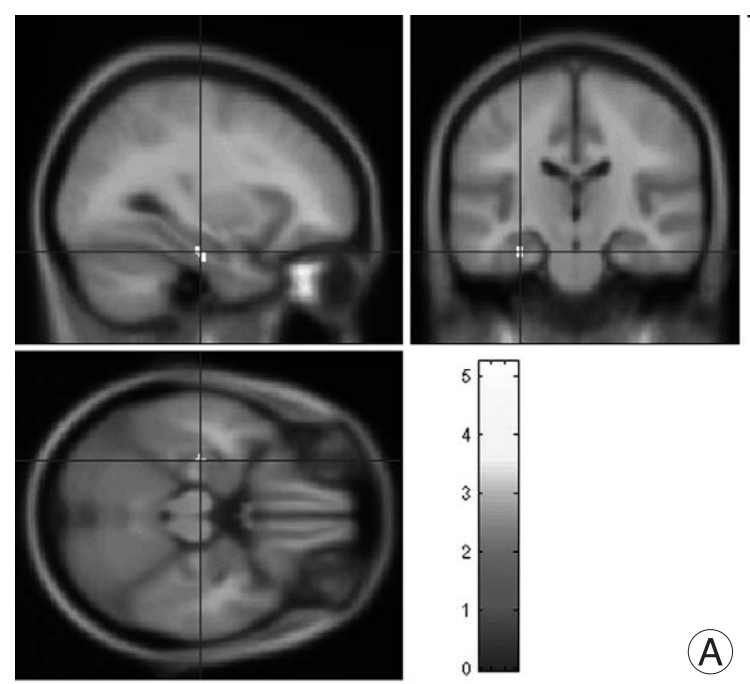

(A)
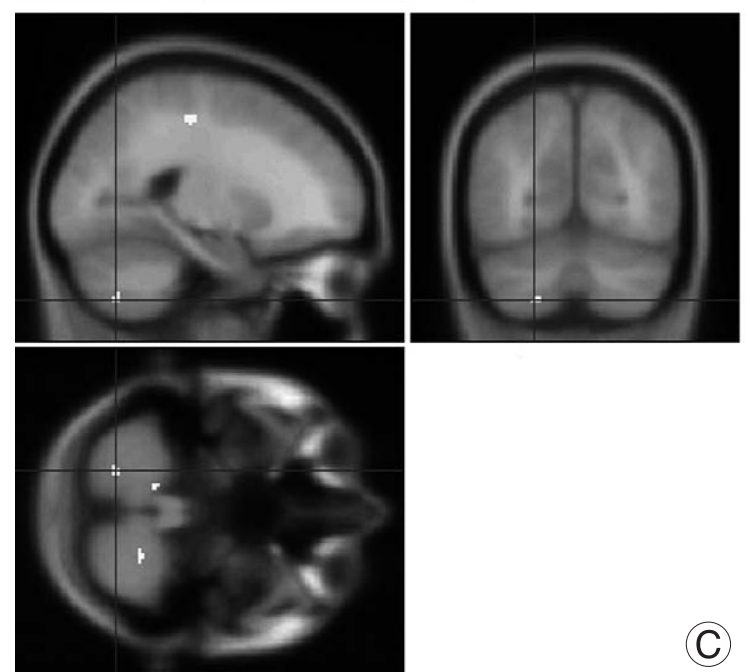
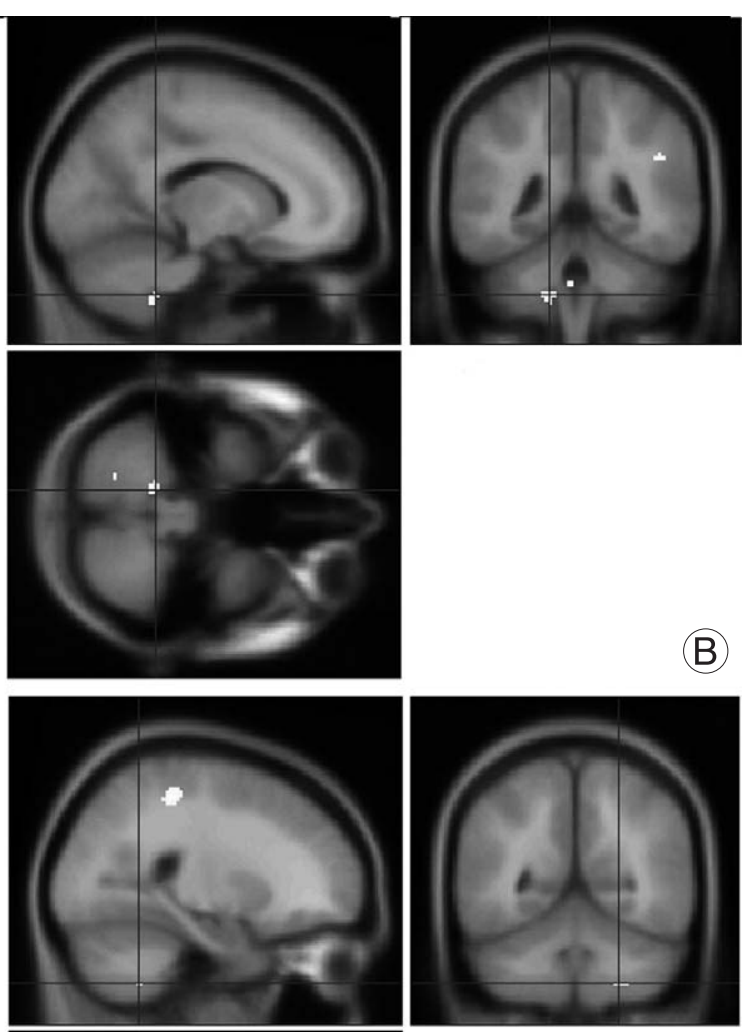

(B)

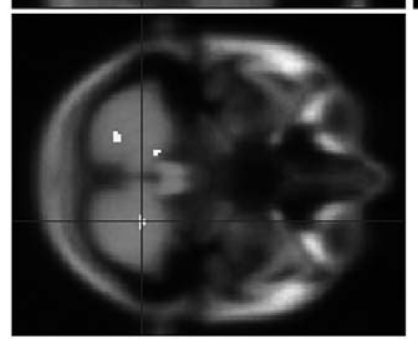

(D)

Fig. 1. Activated brain areas on a follow-up F-18 fluorodeoxyglucose positron emission tomography study in spinal stenosis patients that had preoperative anxiety. (A) Left parahippocampus. (B) Left tonsil. (C) Left inferior semilunar lobule. (D) Right tonsil. 
MR image template provided by SPM2, thereby allowing anatomic identification. To define the effect of treatment on cerebral glucose metabolism between preoperative and postoperative changes, paired $t$-tests were used, which are statistical models in SPM2 based on the general linear model. Also, linear correlations of regional brain glucose metabolism of preoperative and postoperative F-18 FDG PET images with HARS and ZAS scores were investigated using 'single subject: covariates only' analysis and simple regression analysis, statistical models in SPM2 based on the general linear model.

We looked for all voxels where regional cerebral glucose metabolism was significantly correlated with HARS and ZAS scores. A spatial extent threshold of 0 voxels was also used at all times to define any subtle changes in the study group.

\section{Statistical analysis}

Statistical analysis was done with SPSS ver. 11.5 (SPSS Inc., Chicago, IL, USA). Data were expressed as mean ( \pm standard deviation). Preoperative and postoperative data were compared using a paired $t$-test. A $p<0.05$ was regarded as statistically significant.

\section{Results}

\section{Patient characteristics}

There were 18 SS patients ( 13 women and 5 men) with a mean age of $64.7 \pm 8.2$ years in this study. The mean preoperative HARS score was $31.3 \pm 4.9$ and the mean preoperative ZAS score was $51.8 \pm 4.1$. The mean postoperative HARS score at 6 months was $12.3 \pm 3.7$ and the mean postoperative ZAS score at 6 months was $28.8 \pm 4.0$. The mean HARS score and ZAS score were significantly lower postoperatively than preoperatively $(p<0.001$ and $p<$ 0.001 , respectively).

\section{Brain areas activated postoperatively}

Comparison of pre and postoperative F-18 FDG PET scans showed activated brain areas in several brain clusters in gray matter (Table 1, Fig. 1). These included left parahippocampus (voxel size 12, peak $\mathrm{Z}$ vale $=3.4, p<0.001$ ), left cerebellar tonsil (voxel size 20, peak $\mathrm{Z}$ value $=3.35, p<$ 0.001 ), left inferior semi-lunar lobule (voxel size 11, peak $Z$ value $=3.32, p<0.001$ ), and right cerebellar tonsil (voxel size 6 , peak $Z$ value $=3.21, p=0.001$ ).

\section{Postoperative deactivated brain areas}

Table 2 and Fig. 2 show deactivated brain areas postoperatively in SS patients that had preoperative anxiety. Brain areas included are right insula (voxel size 114, peak $\mathrm{Z}$ value $=4.2, p<0.001)$ (Brodmann area 13), left fusiform gyrus $($ voxel size 324 , peak $\mathrm{Z}$ value $=4.07, p<0.001)($ Brodmann area 37), left orbitofrontal cortex (voxel size 37, peak $Z$ value $=3.48, p<0.001)$ (Brodmann area 10), left inferior frontal gyrus (voxel size 12 , peak $\mathrm{Z}$ value $=3.42, p<0.001$ ) (Brodmann area 9), left middle frontal gyrus (voxel size 2, peak $\mathrm{Z}$ value $=3.32, p<0.001)$ (Brodmann area 8$)$, left precuneus (voxel size 4 , peak $\mathrm{Z}$ value $=3.30, p<0.001$ ) (Brodmann area 31), and left inferior frontal gyrus (voxel size 6 , peak $Z$ value $=3.18, p=0.001)($ Brodmann area 9)

\section{Correlation of brain metabolism and anxiety scores}

No significant correlation was found between (a) HARS

Table 2. Deactivated brain areas at follow-up F-18 FDG PET study in spinal stenosis patients with preoperative anxiety

\begin{tabular}{|c|c|c|c|c|c|c|c|c|}
\hline \multirow[b]{2}{*}{ Cluster size } & \multirow[b]{2}{*}{ Hemisphere } & \multicolumn{3}{|c|}{ Coordinates } & \multirow[b]{2}{*}{ Peak Z } & \multirow[b]{2}{*}{$p$-value } & \multirow[b]{2}{*}{ Structure } & \multirow[b]{2}{*}{$\mathrm{BA}$} \\
\hline & & $\mathrm{X}$ & $\mathrm{Y}$ & $\mathrm{Z}$ & & & & \\
\hline 324 & Left & -50 & -60 & -16 & 4.07 & $<0.001$ & Fusiform gyrus & 37 \\
\hline 114 & Right & 36 & 22 & 2 & 5.69 & $<0.001$ & Insula & 13 \\
\hline 37 & Left & -26 & 60 & 24 & 3.48 & $<0.001$ & Orbitofrontal cortex & 10 \\
\hline 12 & Left & -50 & 0 & 24 & 3.42 & $<0.001$ & Inferior frontal gyrus & 9 \\
\hline 6 & Left & -36 & 4 & 32 & 3.18 & 0.001 & Inferior frontal gyrus & 9 \\
\hline 4 & Left & -12 & -46 & 38 & 3.3 & 0.001 & Precuneus & 31 \\
\hline 2 & Left & -20 & 26 & 44 & 3.32 & 0.001 & Middle frontal gyrus & 8 \\
\hline
\end{tabular}

F-18 FDG PET: F-18 fluorodeoxyglucose positron emission tomography, BA: Brodmann area. 

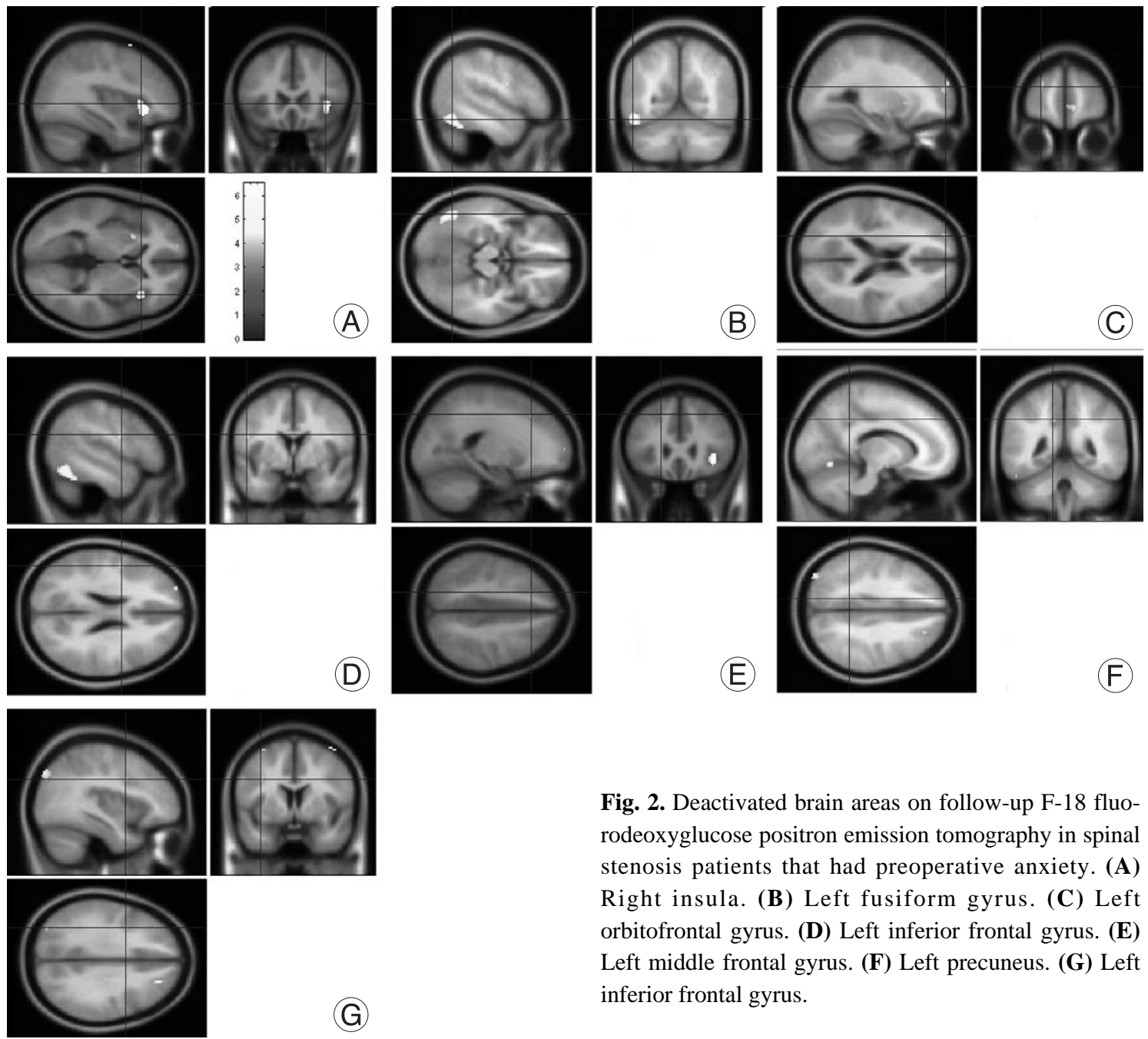

(E)
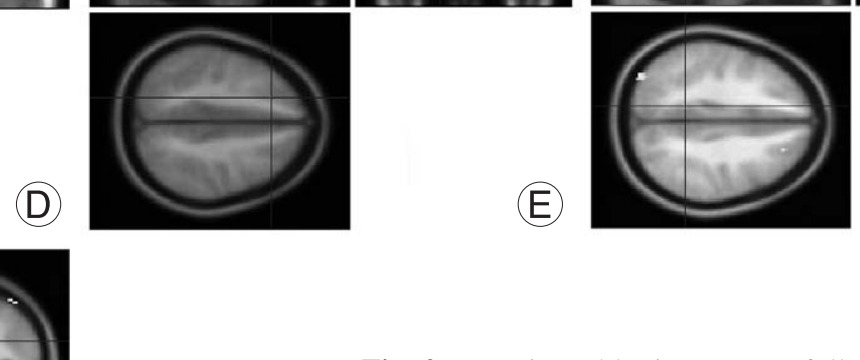

Fig. 2. Deactivated brain areas on follow-up F-18 fluorodeoxyglucose positron emission tomography in spinal stenosis patients that had preoperative anxiety. (A) Right insula. (B) Left fusiform gyrus. (C) Left orbitofrontal gyrus. (D) Left inferior frontal gyrus. (E) Left middle frontal gyrus. (F) Left precuneus. (G) Left inferior frontal gyrus.

or ZAS scores and (b) abnormalities in cerebral glucose metabolism in any preoperative or postoperative F-18 FDG PET images.

\section{Discussion}

The current study shows that several brain areas are postoperatively activated after surgical treatment in SS patients that had preoperative anxiety. During follow-up, SS patients that had preoperative anxiety showed increased cerebral glucose metabolism in several brain areas including both hemispheric cerebellar tonsils and left parahippocampus. Recently, the relationship between cerebellar regions and panic disorder was reported, which suggests a role for the cerebellum in fear-conditioning consolidation [12]. Similar results were found in a recent study [7]. In that study, panic disorder patients showed appreciably greater state anxiety before scanning, and exhibited significantly higher levels of glucose uptake in the bilateral amygdala, hippocampus, and thalamus, and in the midbrain, caudal pons, medulla, and cerebellum than controls.

The parahippocampus is believed to be associated with increased regional cerebral blood flow and metabolism in subjects with panic disorders [8]. In an F-18 FDG PET study, the severity of anxiety symptoms in affective disorder patients was reported to be positively correlated with regional cerebral glucose metabolism in the right parahippocampal and left anterior cingulate regions [13].

In the current study, some deactivated brain areas were noted on the follow-up F-18 FDG PET study. Among these deactivated brain areas, the fusiform gyrus is considered to be associated with a part of the higher sensory cortex that is involved in the perceptual representation of social stimuli [14]. A recent study showed that the right fusiform gyrus and the right lingual gyrus showed a strong negative correlation between the total Liebowitz Social Anxiety Scale 
score and resting brain perfusion in patients with social anxiety disorder [15]. These brain areas may point to defective perception of self and others in social anxiety disorder patients [15].

Previous neuroimaging studies found that panic disorder patients either at rest or during panic attacks had altered function in limbic and paralimbic structures such as the parahippocampal gyrus, ventromedial prefrontal cortex, anterior cingulate cortex, insula, hippocampus, amygdala, brain stem and cerebellum [7,16-18]. Among these limbic and paralimbic structures, the insula also has a distinctive role in the panic attack. In social anxiety disorder patients, the insula showed increased activity during anticipatory anxiety and during a fear conditioned response [19]. However, during a fear conditioned public speaking task, the insula showed decreased activity [20]. In particular, the insular cortex is supposed to process visceral responses accessible to awareness as subjective feeling states [21]. Some studies have found altered insular function in anxiety disorders. It has been shown that the insula is activated during anticipatory anxiety paradigms in healthy subjects and in patients with social anxiety disorders $[19,22]$.

Similar to the current study, Warwick et al. [15] reported that effective pharmacotherapy decreases resting insular perfusion bilaterally in social anxiety disorder patients. They speculated that resting brain perfusion may be increased in the insula in social anxiety disorder patients, with normalization following successful pharmacotherapy. Kilts et al. [23] also demonstrated that the insula shows increased activity related to re-experiencing social anxiety provoking situations after nefazodone treatment.

Interestingly, in the current study, the orbitofrontal cortex, and the inferior and middle frontal cortex showed deactivation during a follow-up F-18 FDG PET study in SS patients that had preoperative anxiety. The orbitofrontal cortex has been implicated in a variety of functions, particularly higher-order executive functions. The orbitofrontal cortex and amygdala are supposed to be components of the neural circuitry involved in the adaptive processing of emotion and in psychopathology [24]. A recent study demonstrated that orbitofrontal cortex lesions significantly increased left frontal asymmetric brain electrical activity, which is a finding similar to findings in the current study [25]. Recurrent hyperperfusion in the right anterior lateral orbitofrontal cortex was also reported recently in obsessivecompulsive disorder patients and this finding provides evidence of orbitofrontal cortex involvement in the pathophys- iology of obsessive-compulsive disorder [26]. Contrary to the current and previous reports, right orbitofrontal cortex gray matter volume was significantly smaller in breast cancer survivors with post-traumatic stress disorder (PTSD) than in those without PTSD or healthy subjects [27]. They concluded that the orbitofrontal cortex, which is involved in the extinction of fear conditioning or the emotional retrieval of autobiographical memory, forms part of the neurobiological basis of PTSD.

Using PET, a study found that changes in brain metabolism after treatment with either cognitive behavioral therapy or antidepressants were similar in several brain areas: decreases were found in the right hemisphere, in superior, middle and inferior frontal gyrus, and superior and middle temporal gyrus; increases were detected in the left hemisphere, inferior frontal gyrus, superior, middle and transverse temporal gyrus, and insula. However, no changes were found in limbic areas [18].

The right precuneus was reported to show decreased resting brain perfusion in social anxiety disorder patients compared to healthy controls in a recent study [15]. According to a previous study, social anxiety patients had right precuneus activation in response to a social task and an evaluation task following pharmacotherapy [23]. Similar to the current study, Carey et al. [28] reported that the left precuneus showed deactivation in obsessive-compulsive disorder responders relative to non-responders following treatment with inositol [29].

However, the current study could not find any evidence for excessive amygdalo-hippocampal activity and changes of glucose metabolism in the same circuit at follow-up in SS patients that had preoperative anxiety.

It is unclear whether different neurocircuitry systems are involved in general anxiety disorder and preoperative anxiety disorder. However, modern brain imaging techniques have proved useful in exposing overlapping neurocircuitry that underlies individual anxiety disorders [30].

\section{Conclusions}

SS patients with preoperative anxiety showed altered cerebral glucose metabolism at follow-up in F-18 FDG PET studies. Both cerebellar tonsils, left parahippocampus, and left cerebellar inferior semi-lunar lobule were activated after treatment. Deactivated brain areas include the right insula, left fusiform gyrus, left orbitofrontal cortex, left inferior frontal gyrus, left middle frontal gyrus, left pre- 
cuneus, and left inferior frontal gyrus.

\section{Acknowledgements}

This study was supported by Pusan National University Hospital Research Grant of 2010 year.

\section{REFERENCES}

1. Cooke M, Chaboyer W, Schluter P, Hiratos M. The effect of music on preoperative anxiety in day surgery. J Adv Nurs 2005;52:47-55.

2. de Groot KI, Boeke S, van den Berge HJ, Duivenvoorden HJ, Bonke B, Passchier J. The influence of psychological variables on postoperative anxiety and physical complaints in patients undergoing lumbar surgery. Pain 1997;69:1925.

3. Etkin A, Wager TD. Functional neuroimaging of anxiety: a meta-analysis of emotional processing in PTSD, social anxiety disorder, and specific phobia. Am J Psychiatry 2007; 164:1476-88.

4. LeDoux JE. Emotion circuits in the brain. Annu Rev Neurosci 2000;23:155-84.

5. Phelps EA, Delgado MR, Nearing KI, LeDoux JE. Extinction learning in humans: role of the amygdala and vmPFC. Neuron 2004;43:897-905.

6. Milad MR, Quirk GJ, Pitman RK, Orr SP, Fischl B, Rauch SL. A role for the human dorsal anterior cingulate cortex in fear expression. Biol Psychiatry 2007;62:1191-4.

7. Sakai Y, Kumano H, Nishikawa M, et al. Cerebral glucose metabolism associated with a fear network in panic disorder. Neuroreport 2005;16:927-31.

8. Nordahl TE, Semple WE, Gross M, et al. Cerebral glucose metabolic differences in patients with panic disorder. Neuropsychopharmacology 1990;3:261-72.

9. Nordahl TE, Stein MB, Benkelfat C, et al. Regional cerebral metabolic asymmetries replicated in an independent group of patients with panic disorders. Biol Psychiatry 1998;44:998-1006.

10. Riskind JH, Beck AT, Brown G, Steer RA. Taking the measure of anxiety and depression: validity of the reconstructed Hamilton scales. J Nerv Ment Dis 1987;175:474-9.

11. Zung WW. A rating instrument for anxiety disorders. Psychosomatics 1971;12:371-9.

12. Sacchetti B, Baldi E, Lorenzini CA, Bucherelli C. Cerebellar role in fear-conditioning consolidation. Proc Natl Acad Sci U S A 2002;99:8406-11.
13. Osuch EA, Ketter TA, Kimbrell TA, et al. Regional cerebral metabolism associated with anxiety symptoms in affective disorder patients. Biol Psychiatry 2000;48:10203.

14. Adolphs R. Cognitive neuroscience of human social behaviour. Nat Rev Neurosci 2003;4:165-78.

15. Warwick JM, Carey P, Jordaan GP, Dupont P, Stein DJ. Resting brain perfusion in social anxiety disorder: a voxelwise whole brain comparison with healthy control subjects. Prog Neuropsychopharmacol Biol Psychiatry 2008;32: 1251-6.

16. Bremner JD, Innis RB, White T, et al. SPECT [I-123] iomazenil measurement of the benzodiazepine receptor in panic disorder. Biol Psychiatry 2000;47:96-106.

17. Bystritsky A, Pontillo D, Powers M, Sabb FW, Craske MG, Bookheimer SY. Functional MRI changes during panic anticipation and imagery exposure. Neuroreport 2001;12:3953-7.

18. Prasko J, Horácek J, Záleský R, et al. The change of regional brain metabolism (18FDG PET) in panic disorder during the treatment with cognitive behavioral therapy or antidepressants. Neuro Endocrinol Lett 2004;25:340-8.

19. Lorberbaum JP, Kose S, Johnson MR, et al. Neural correlates of speech anticipatory anxiety in generalized social phobia. Neuroreport 2004;15:2701-5.

20. Tillfors M, Furmark T, Marteinsdottir I, Fredrikson M. Cerebral blood flow during anticipation of public speaking in social phobia: a PET study. Biol Psychiatry 2002;52: 1113-9.

21. Critchley HD, Wiens S, Rotshtein P, Ohman A, Dolan RJ. Neural systems supporting interoceptive awareness. Nat Neurosci 2004;7:189-95.

22. Simmons A, Matthews SC, Stein MB, Paulus MP. Anticipation of emotionally aversive visual stimuli activates right insula. Neuroreport 2004;15:2261-5.

23. Kilts CD, Kelsey JE, Knight B, et al. The neural correlates of social anxiety disorder and response to pharmacotherapy. Neuropsychopharmacology 2006;31:2243-53.

24. Davidson RJ, Lewis DA, Alloy LB, et al. Neural and behavioral substrates of mood and mood regulation. Biol Psychiatry 2002;52:478-502.

25. Kalin NH, Shelton SE, Davidson RJ. Role of the primate orbitofrontal cortex in mediating anxious temperament. Biol Psychiatry 2007;62:1134-9.

26. Matsumoto R, Nakamae T, Yoshida T, et al. Recurrent hyperperfusion in the right orbitofrontal cortex in obsessive-compulsive disorder. Prog Neuropsychopharmacol 
Biol Psychiatry 2008;32:1082-4.

27. Hakamata Y, Matsuoka Y, Inagaki M, et al. Structure of orbitofrontal cortex and its longitudinal course in cancerrelated post-traumatic stress disorder. Neurosci Res 2007; 59:383-9.

28. Carey PD, Warwick J, Harvey BH, Stein DJ, Seedat S. Single photon emission computed tomography (SPECT) in obsessive-compulsive disorder before and after treatment with inositol. Metab Brain Dis 2004;19:125-34.
29. Carey PD, Warwick J, Niehaus DJ, et al. Single photon emission computed tomography (SPECT) of anxiety disorders before and after treatment with citalopram. BMC Psychiatry 2004;4:30.

30. Lucey JV, Costa DC, Busatto G, et al. Caudate regional cerebral blood flow in obsessive-compulsive disorder, panic disorder and healthy controls on single photon emission computerised tomography. Psychiatry Res 1997;74: 25-33. 